\title{
Load-dependent release limits the processive stepping of the tetrameric Eg5 motor
}

\author{
Mikhail J. Korneev • Stefan Lakämper • \\ Christoph F. Schmidt
}

Received: 27 June 2006 / Revised: 20 December 2006 / Accepted: 2 January 2007 / Published online: 28 February 2007

(C) EBSA 2007

\begin{abstract}
Tetrameric motor proteins of the Kinesin-5 family are essential for eukaryotic cell division. The microscopic mechanism by which Eg5, the vertebrate Kinesin-5, drives bipolar mitotic spindle formation remains unknown. Here we show in optical trapping experiments that full-length $\mathrm{Eg} 5$ moves processively and stepwise along microtubule bundles. Interestingly, the force produced by individual Eg5 motors typically reached only $\sim 2 \mathrm{pN}$, one-third of the stall force of Kinesin-1. Eg5 typically detached from microtubules before stalling. This behavior may reflect a regulatory mechanism important for the role of $\mathrm{Eg} 5$ in the mitotic spindle.
\end{abstract}

\section{Introduction}

Members of the Kinesin-5 family are homo-tetramers with two motor domains at each end of a central fourstranded stalk. Kinesin-5 motors play a crucial role in cell division (Kashina et al. 1996; Sawin et al. 1992). We have recently shown that Eg5 drives relative sliding of two microtubules, suggesting that each dimeric end walks on one microtubule (Kapitein et al. 2005). A recent study using single-molecule fluorescence has

Mikhail J. Korneev and Stefan Lakämper have contributed equally to this work.

M. J. Korneev · S. Lakämper · C. F. Schmidt ( $\)$

Department of Physics and Astronomy, Vrije Universiteit, de Boelelaan 1081, 1081 HV Amsterdam, The Netherlands e-mail: cfs@physik3.gwdg.de

S. Lakämper · C. F. Schmidt

III. Physikalisches Institut, Georg-August-Universität,

Friedrich-Hund-Platz 1, 37077 Göttingen, Germany furthermore revealed that single Eg5 motors can run processively on microtubules and on axonemes (microtubule bundles) (Kwok et al. 2006). Processivity has also been reported for a truncated dimeric construct (Valentine et al. 2006), in contrast to earlier reports of non-processivity also of a dimeric construct in kinetic experiments (Crevel et al. 1997).

In vivo, $\mathrm{Eg} 5$ is believed to drive the poleward sliding of microtubules during spindle morphogenesis (Miyamoto et al. 2004). Eg5 thus works in a large organized machinery which resembles muscle in its function if not in its microstructure (Sharp et al. 2000). In contrast to muscle, the structural elements in the spindle are microtubule bundles which are rigid enough to withstand compressive forces. Since motors of opposite directionalities are active in the spindle, the whole assembly works like a "push-pull muscle" (Hildebrandt and Hoyt 2000; Sharp et al. 2000). The competition between opposing motors might serve as one regulatory element in this highly complex system. During spindle morphogenesis, the spindle poles move apart to a well defined distance which is then maintained in a dynamic equilibrium while the poleward flux of microtubules continues (Miyamoto et al. 2004). This equilibrium could be controlled by a length measurement (e.g. via chemical gradients). It is tempting, however, to speculate that this equilibrium, just as the positioning of the chromosomes in the cell midplane in metaphase, is maintained through a balance of forces. In that case it is necessary for the cell to sense force in some way, possibly using the motors themselves which could act as force sensors via load-dependent steps in their chemical cycles. Such a mechanism has been proposed to explain spindle oscillations in asymmetric cell division in C. elegans (Grill et al. 2005). 
Understanding the exact role of $\mathrm{Eg} 5$ in the mitotic spindle thus requires a measurement of the force generated by the full-length motor beyond just observing its unloaded motion on the microtubule lattice.

\section{Materials and methods}

\section{Protein preparation}

Full-length Xenopus laevis Eg5 with an amino-terminal poly-histidine tag was expressed in insect cells, purified as described with an additional purification step using gel filtration on a Superose 6 column (Kapoor and Mitchison 2001) and stored at $-80^{\circ} \mathrm{C}$. We used an amino-terminal tag in order to avoid a possible perturbation of the interaction of the strongly conserved BimC box at the C-terminus of the motor with a head of the opposing dimer. In addition we wanted to provide an easily accessible poly-histidine epitope for antibody immobilization of the motor. His-tagged Eg5 for functionality in a rescue assay in Xenopus egg extract and no difference to untagged motors was found (Kwok et al. 2004). Axonemes were purified from sea urchin sperm following a published protocol (Gibbons and Fronk 1979). C-terminal Histagged Drosophila melanogaster kinesin heavy chain (DmKHC) truncated at amino acid 685 was purified by using immobilized metal affinity chromatography (IMAC). E. coli strain BL21(DE3) was used to overexpress kinesin and the cells were lysed by a freeze/ thaw method. The cell lysate was then mixed with Ni-NTA resin, the column was washed with wash buffer, and kinesin was eluted from the column and stored at $-80^{\circ} \mathrm{C}$.

\section{Optical trapping microscope}

Assays were performed at $21^{\circ} \mathrm{C}$ using a single-beam optical trap setup built on a custom-designed inverted microscope as described elsewhere (Allersma et al. 1998). Infrared laser light $\left(1,064 \mathrm{~nm}, \mathrm{cw}, \mathrm{Nd}: \mathrm{YVO}_{4}\right.$, Compass, Coherent, Santa Clara, CA, USA) was focused into the flow chamber using an objective lens (Neofluar $100 \times$, 1.3 NA, oil immersion, Zeiss) to trap the particle. The trap stiffness was varied in the range of $1-5 \times 10^{-5} \mathrm{~N} \mathrm{~m}^{-1}$. The back-focal plane of the condenser (1.4 NA, oil immersion, Zeiss) was imaged onto a quadrant photodiode, which was operated at a reverse bias voltage of $100 \mathrm{~V}$ (YAG444-4A, Perkin Elmer, Vaudreuil, Canada) for position detection of the trapped particle (Gittes and Schmidt 1998a). Photodiode signals, reflecting the displacement fluctuations of the trapped particle in the plane normal to the optical axis, were processed by custom-built analog electronics (Allersma et al. 1998) and read into a PC using LabView (National Instruments, Austin, TX, USA) for digital processing.

\section{Sample preparation}

Motility assays were carried out in sample chambers that were assembled from a cover slip and microscope slide, separated by a double-stick tape with a thickness of $\sim 70 \mu \mathrm{m}$. Streptavidin-coated silica particles $0.5 \mu \mathrm{m}$ in diameter (Kisker Biotech, Germany) were incubated first with $0.09 \mathrm{mg} \mathrm{ml}^{-1}$ casein (Sigma-Aldrich) in PEM80 buffer (80 mM PIPES, $1 \mathrm{mM} \mathrm{MgCl}_{2}, 1 \mathrm{mM}$ EGTA, pH 6.8) for 10 min prior to mixing with biotinylated antibodies against the poly-histidine tag (PentaHis Biotin Conjugate, Qiagen) and then incubated further for at least $0.5 \mathrm{~h}$. In order to eliminate unbound antibodies the beads were washed three times in PEM80 buffer containing $90 \mu \mathrm{g} \mathrm{ml}^{-1}$ casein by centrifugation (10 min, 6,000 rpm, Centrifuge 5415D, Eppendorf) and resuspension, before motors were added. Then Eg5 was added in the presence of $1 \mathrm{mM}$ ATP in PEM80, and the mixture was incubated for $30 \mathrm{~min}$ on ice. Axonemes in PEM80 were injected into a flow chamber and incubated for $10 \mathrm{~min}$ to let the filaments bind to the surface. To prevent non-specific bead binding, the flow chamber was then treated with $0.09 \mathrm{mg} \mathrm{ml}^{-1}$ casein for another $10 \mathrm{~min}$. In order to remove unbound axonemes, the flow chamber was washed with several volumes of motility buffer consisting of PEM80, $10 \mathrm{mM}$ dithiothreitol, $1 \mathrm{mM}$ ATP, $2 \mathrm{mM} \mathrm{MgCl}_{2}, 10 \mathrm{mM}$ glucose, $100 \mu \mathrm{g} \mathrm{ml}^{-1}$ glucose oxidase, $80 \mu \mathrm{g} \mathrm{ml}^{-1}$ catalase, $90 \mu \mathrm{g} \mathrm{ml}^{-1}$ casein. Finally, the mixture of motility buffer, which contained motorcoated beads with antibodies at a concentration of 0.06 $0.13 \mu \mathrm{g} \mathrm{ml}^{-1}$ and Eg5 at a concentration of $1-4 \mu \mathrm{g} \mathrm{ml}{ }^{-1}$, was added.

Single-molecule motility experiments

Axonemes were chosen with their long axis oriented parallel to one of the quadrant photodiode detector axes. Using a piezo-actuated XYZ substage (Nano-LP100, Mad City Labs, Madison, USA), the laser-trapped motor-coated bead was positioned on top of a surfaceimmobilized filament to allow the motors on the surface of the trapped bead to interact with the microtubule lattice. The position of the bead in the trap was monitored using a quadrant photodiode detector. The detector output was sampled at $4,096 \mathrm{~Hz}$. Position measurements were calibrated using the power spectrum method (Gittes and Schmidt 1998b). We estimate 
an error of $\sim 20 \%$ in this calibration, which is taken into account in calculating the error of the motor velocity. Data were acquired, stored and analyzed using custom-written software in LabView.

\section{Results and discussion}

To explore Eg5's motility with high spatial and temporal resolution and under load, we have used a single-bead optical trapping motility assay. We have used biotinylated antibodies to the His-tags at the N-termini of fulllength $\mathrm{Eg} 5$ to attach the motors to streptavidin-coated silica spheres. For a tetrameric double-ended motor, attachment to the bead can occur in different geometries, and the probability of binding in a particular geometry depends on stoichiometry, namely on the ratio of motors to beads and that of motors to antibodies. The different conceivable binding modes are sketched in Fig. 1. Only two of these binding configurations (d and f) are expected to allow unperturbed microtubule interactions by two motor domains at one

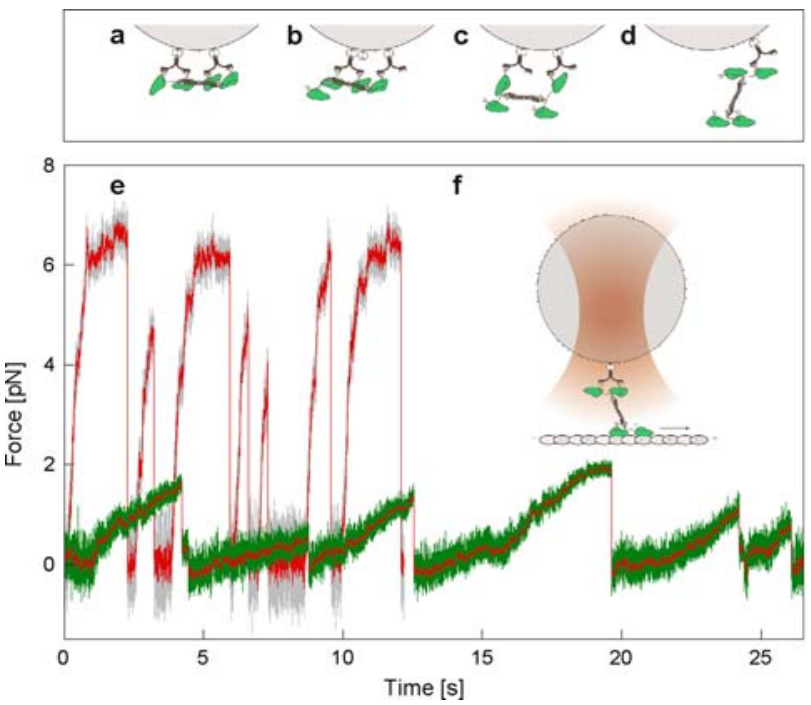

Fig. 1 Motor attachment, experimental setup and bead traces. ad, f Possible Eg5-motor attachments to beads (silica, $0.5 \mu \mathrm{m}$ diameter) via the genetically encoded N-terminal His-tag of Eg5. a All four motor domains are bound, preventing motility. b One motor domain is unbound, likely allowing only non-processive motility. c Two motor domains are free, one at each end, likely allowing only non-processive motility. d One motor domain is bound, leaving a dimeric motor end free to interact with the microtubule. $\mathbf{e}$ Traces of bead motility generated by individual Eg5 (green) and Kinesin-1 (grey) motors. The averaged (15-point) and median-filtered (0.3 s (Eg5) and $0.05 \mathrm{~s}$ (Kinesin-1) sliding windows; rank 10) signal is overlaid in red over both traces. The trap stiffnesses were $0.03 \mathrm{pN} / \mathrm{nm}$ (Kinesin-1) and $0.013 \mathrm{pN} / \mathrm{nm}(\mathrm{Eg} 5)$. f One dimer is bound, one dimer is free; sketch of a silica-sphere with motor held in the laser-trap, such that it interacts with a surface-attached microtubule track end of the tetramer. To achieve binding in these configurations, we optimized the relative concentrations of motors to antibodies and beads by determining the fractions of beads binding and moving on surfaceattached axonemes (Fig. 2a, b). At a high antibody density on the beads (Fig. 2a), less beads moved than were stuck or bound only briefly, even when the motor concentration was increased. This might have been caused by mutual inhibition of tightly packed motors. At the lowest motor density no binding or motility was observed, most likely because all motor-domains were sequestered by the excess of antibody binding sites. At a constant motor concentration in solution (Fig. 2b), the fraction of interacting and motile beads first increased with increasing antibody concentration, reached a maximum at a ratio of about 2:1 (motors:antibodies) and then decreased again. This again clearly demonstrates that an excess of antibodies is likely to bind the motors in a non-functional geometry.

At the optimal motor-to-antibody ratio (between 1:1 and 2:1) we then reduced the absolute concentrations of both (Fig. 2c). As expected, the fraction of beads moving on microtubules decreased. Assuming Poisson binding statistics (Svoboda and Block 1994; Svoboda et al. 1993), the likelihood is large that the observed bead-microtubule-interactions are driven by just one motor if the fraction of moving beads is below $50 \%$. For all our subsequent single-motor experiments, a motor/antibody ratio of 2 and absolute densities of nominally $\sim 200$ motors/bead were used. This is an upper limit for the motor density on the beads, assuming that all motors were functional and did bind to beads. Since in reality this will not be the case, this nominal density is reasonable.

We recorded the movement of Eg5-bead complexes along axonemes with high-resolution back-focal-plane interferometry (Gittes and Schmidt 1998a). Beads moved processively at an average rate of $35 \pm 8 \mathrm{~nm} / \mathrm{s}$ (Fig. 1e), consistent with velocities observed in multiple-motor surface-gliding assays (data not shown). As a control, we used Kinesin-1 (DmKHC685, the plasmid was a kind gift from R.J. Stewart, University of Utah) with a C-terminal His-tag which allowed us to attach it to the same kind of beads in the same way. Kinesin-1coated beads moved with initial speeds of about 400 $600 \mathrm{~nm} / \mathrm{s}$ and typically stalled in the optical trap at loads of 6-7 pN (Fig. 1e) consistent with what has generally been found for Kinesin-1's (Svoboda and Block 1994; Svoboda et al. 1993). As shown in Fig. 1e, the traces of Eg5-driven beads reveal a very distinct behavior. Apart from the fact that the velocity was much lower than that of kinesin, motor-driven excursions 


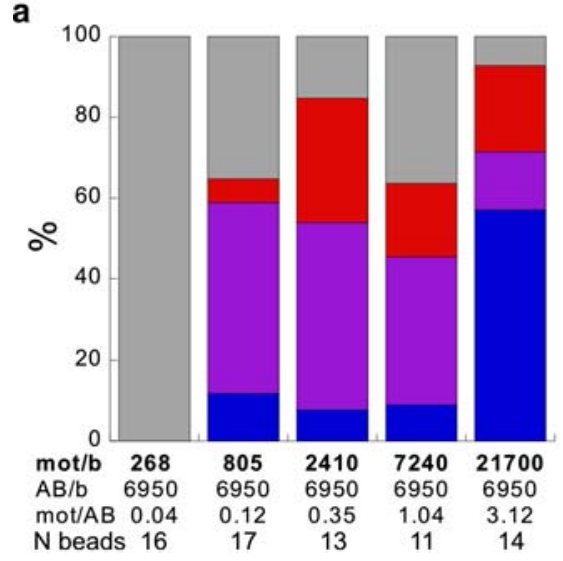

b

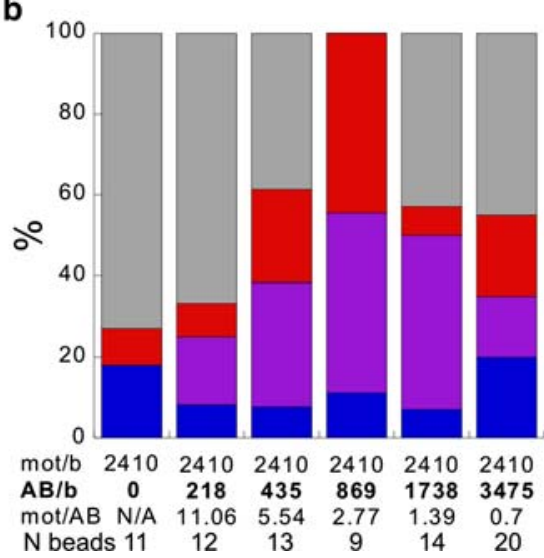

C

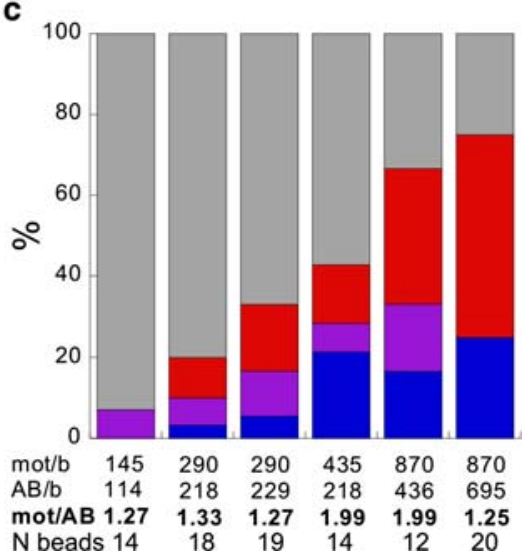

Fig. 2 Motor-bead attachment. Fractions of beads not interacting with a microtubule over 2 min (grey), moving (red), attached for less than 2 min (without motion) (purple), irreversibly stuck (blue). a Constant high average number of antibodies per bead $(6,950)$, variation of motor concentration. b Constant concentration of motors in solution (corresponding to 2,410 per bead), variation of antibody density in order to obtain optimal motor/

from the center of the trap appeared less regular. The thermal motion of the beads clearly decreased with increasing load, reflecting the non-linearity of the attachment compliance (Svoboda et al. 1993). At a trap stiffness $\kappa_{\text {trap }}=0.013 \mathrm{pN} / \mathrm{nm}$, the average displacement from the trap center reached only about $100 \mathrm{~nm}$ and the load at which motors detached was typically below $2 \mathrm{pN}$. The repeated excursions observed, even at low motor concentrations, make it unlikely that the release events were caused by the breakage of the antibodypoly-his bond. The same bond survived a much higher force in the kinesin experiments. Moreover, motor beads bound to axonemes in the presence of the nonhydrolyzable ATP analog AMP-PNP withstood much higher forces (data not shown).

To examine the motility mechanism more closely, we first analyzed the motility records of Kinesin-1 for evidence of regular stepping. At limiting motor concentrations, beads moved in the stereotypical kinesin pattern (Fig. 3). At higher forces the overall stiffness of the system was increased sufficiently to readily observe stepwise displacements.

We then analyzed Eg5 motility in the same way. Figure 4a shows a collection of Eg5-microtubule interaction events in which stepwise motion was visible. Due to the low load levels Eg5 can sustain, the stiffness of the axoneme-bead-linkage was not in all traces high enough to show steps. The pairwise-distance distribution functions (PDFs) (Svoboda et al. 1993) of the plotted interactions, however, that were selected for more rigid attachment, revealed step-wise motion driven by individual Eg5-motors (Fig. 4b). Note that the initial antibody-ratio ( $r$-value) to favor species $\mathbf{d}$ and $\mathbf{f}$ (Fig. 1). c Systematic reduction of the absolute number of motor/antibody-complexes at constant $r$-values between 1 and 2 revealed a clear concentration dependence of motile events. Data were collected typically over periods of minutes. The number of beads that was used at each concentration is shown under the histograms

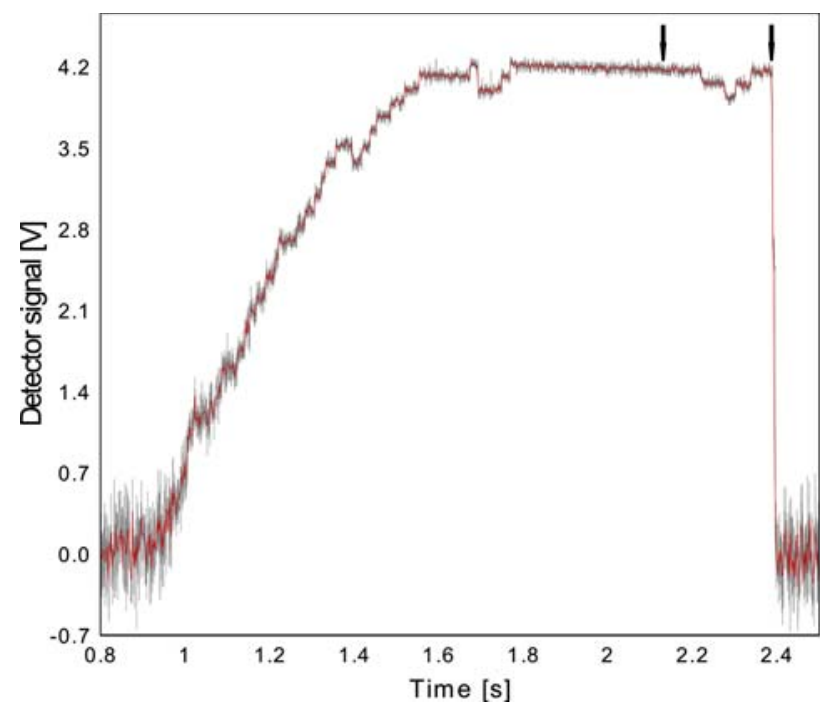

Fig. 3 Steps of Kinesin-1/control. Kinesin-1 motility using the same attachment chemistry to immobilize individual motors on $0.5 \mu \mathrm{m}$ silica spheres. Stepwise displacement (uncalibrated axis) is evident before (grey) and after filtering the data (red, $4,096 \mathrm{~Hz}$ sampling rate, 15-point averaging and median-filtering: $0.05 \mathrm{~s}$ sliding window, rank 10)

variance shown in the traces does not strictly scale with trap stiffness because from track to track varying linkage stiffness dominated in most cases. The measured step-size $\quad \Delta_{\text {bead }}=6.5 \pm 0.2 \mathrm{~nm} \quad($ mean \pm SEM) was attenuated by the compliance of the axoneme-surfaceand motor-bead linkages and is thus smaller than $8 \mathrm{~nm}$. It is possible to roughly determine the magnitude of this attenuation factor. From the displacement variance in the tracks shown in Fig. 4a we estimate an average 

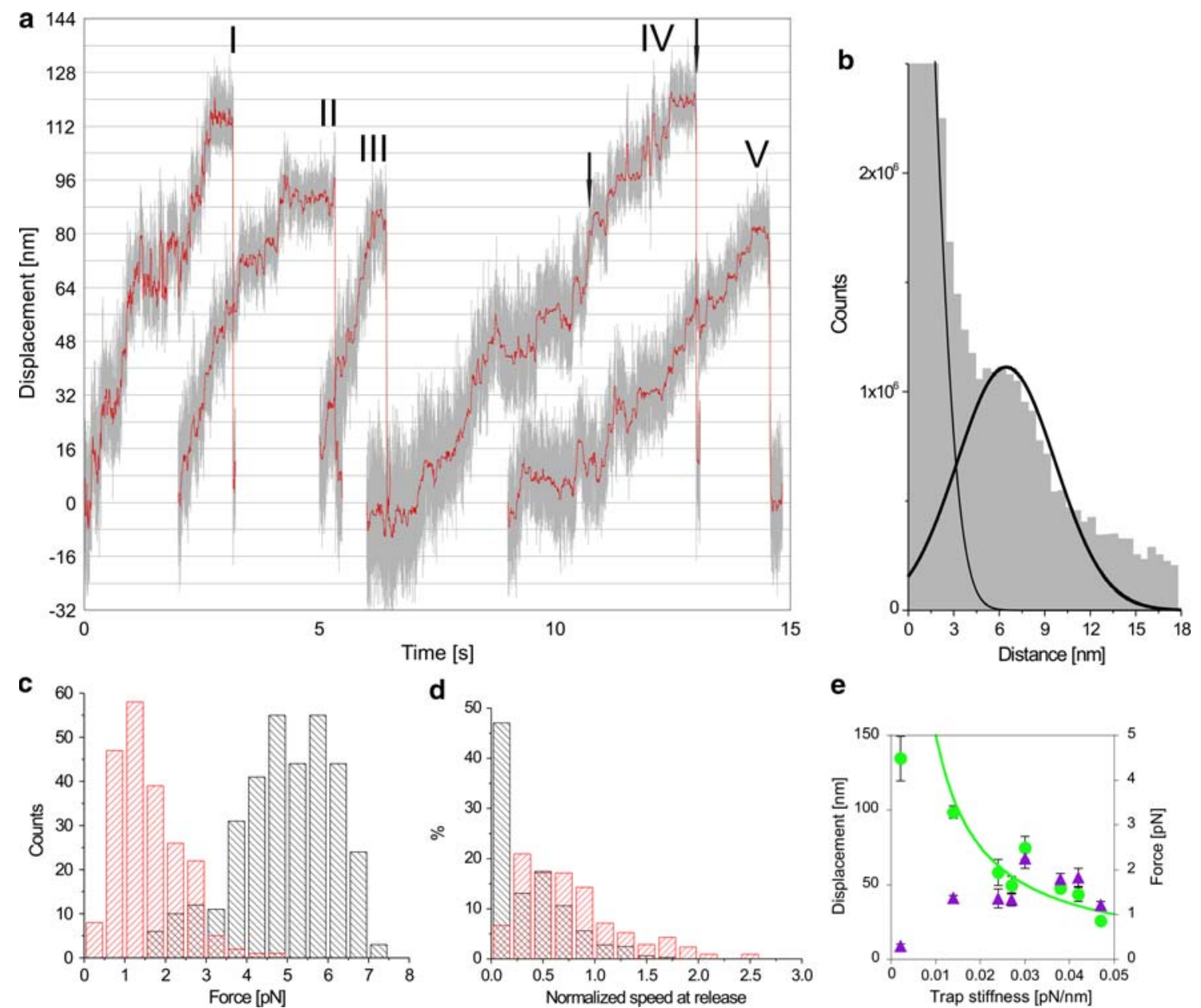

e

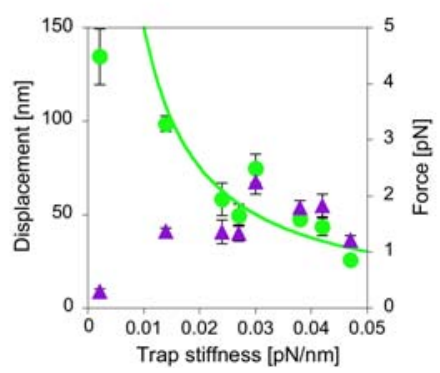

Fig. 4 Single-step analysis and quantification of release force. a Example traces selected from independent experiments displaying stepwise displacements of the beads in the laser-trap driven by individual Eg5 motors [grey, unfiltered data; red, averaged (15point) and median-filtered data (0.3 s sliding window, rank 10)]. The trap stiffnesses were: trace I: 0.014; II: 0.021; III, IV: 0.029; V: $0.042 \mathrm{pN} / \mathrm{nm}$. b PDF of 10 traces including the ones shown in a to detect steps. Double Gaussian fit to this distribution yields a mean step size of $6.5 \pm 0.2 \mathrm{~nm}$ (mean \pm SEM). c Histogram analysis of detachment forces observed for single Kinesin-1- (black) and Eg5-motors (red). d Histograms of relative motor speeds

linkage stiffness of $\kappa_{\text {link }}=0.062 \mathrm{pN} / \mathrm{nm}$. To estimate the motor displacement $\Delta_{\text {mot }}$ we use the correction $\Delta_{\text {mot }}=\Delta_{\text {bead }}\left(\kappa_{\text {trap }}+\kappa_{\text {link }}\right) / \kappa_{\text {link }}$. This results in a step size of $9.3 \pm 1.5 \mathrm{~nm}$, which is consistent with the $8 \mathrm{~nm}$ periodicity of the tubulin lattice. The linkage stiffness found for Eg5 is comparable to what has been reported for similar experiments with Kinesin-1 (Svoboda and Block 1994; Svoboda et al. 1993). Processive stepping behavior similar to what we see has also recently been found with truncated dimeric human Eg5 constructs (Valentine et al. 2006), although only in high ionic strength buffer $(200 \mathrm{mM} \mathrm{KCl})$ and for short runs, comprising directly preceding detachment: we scored the speeds of beads driven by Kinesin-1 (black) and Eg5 (red) in time interval before detachment (between arrows in a and Fig. 3 corresponding to about 10 motor steps in both cases) relative to the unloaded motors speeds. e Systematic variation of the trap stiffness revealed a hyperbolic relation to the obtained displacement (green circles). Error bars denote SEM, the number of evaluated events varied between $5<N<73$. The resulting detachment forces (product of displacement and trap stiffness) are nearly constant (purple triangles)

only a few steps. Our finding of stepping behavior (typically tens of steps) for the full-length tetrameric motor at standard motility assay conditions (80 mM PIPES) supports the hypothesis that the motor can function in vivo in a processive manner and that each dimeric end of the motor can exert force on a microtubule.

The distinctive feature of full-length Eg5 motility was that the maximal force was on average rather low and that the motor tended to release at higher forces (Fig. 1e). In the light of the stepping data this is unlikely to stem from the alternative attachment geometries (Fig. 1a-c). To quantify the phenomenon, we measured 
the Eg5 release forces in comparison to Kinesin-1 (Fig. 4c). While Kinesin-1 reached on average 5 pN, $\mathrm{Eg} 5$ only reached an average force of $1.6 \mathrm{pN}$. The shapes of the distributions of detachment-forces were also clearly different between the two motors. Kinesin-1 was most likely to release at about $5 \mathrm{pN}$, with a tail in the distribution towards lower forces, while $\mathrm{Eg} 5$ mostly released at low force with a tail towards higher forces. The tail towards higher forces observed with Eg5 (Fig. 4c) could be due to the finite probability of having more than one motor interacting with the microtubule on some beads or of having the two dimers of one tetramer somehow interacting at the same time with the microtubule, thereby producing higher force.

We tested whether detachment occurred before or during stalling by plotting a distribution of speeds immediately preceding detachment, normalized by the average initial speeds (Fig. 4d). While Kinesin-1 predominantly detached at very low relative speed (i.e. during stalling), Eg5-motors dissociated more frequently at near-maximal speeds and rarely stalled completely. To further test for a load-dependent release mechanism, we repeated the experiments with a range of trap stiffnesses. A motor with a load independent run length would not be affected by varying trap stiffness, while a motor with a characteristic release force should release at decreasing displacements with increasing trap stiffnesses. Average release displacements and corresponding forces are plotted in Fig. 4e. We found an almost constant release force (Fig. 4e, triangles) while displacements scaled as the inverse of trap stiffness (Fig. 4e, circles). Note that the first point, both in displacement and force, at low trap stiffness values does not fit on the model curves. This can be explained by another run length-limiting factor taking over from load-triggered release.

Our findings suggest that Eg5 might employ a so far un-described mechanism to limit force-production of individual motors-a sort of slip-clutch-mechanismwhich might have a role in regulating spindle dynamics. It is interesting to note that, in contrast to skeletal muscle, regulation of the spindle dynamics is not effected from the outside, but cell-internally. In the most widely accepted model for spindle formation and maintenance, the "push-pull muscle" model, opposing motors balance each other in a carefully regulated fashion (Compton 2000; Hildebrandt and Hoyt 2000; Sharp et al. 2000). Since Eg5 is believed to be involved in this force balance, the effect we observed would give Eg5 the role of a force sensor that would act such that force can be kept constant at a non-zero velocity. It is unclear if the load-dependent detachment is a property of the motor domain itself or if it depends on the structural organization of the tetrameric motor (neck and/or the coiled-coil). Since a dimeric truncated construct has been shown to stall at higher force (Valentine et al. 2006), it is possible that the tails of the opposing motor dimer and in particular the highly conserved BimC box (Drummond and Hagan 1998) are necessary for this regulation. To clarify this, future studies will have to be done with engineered chimeric and truncated Eg5-constructs. Our study provides insight into the native tetramer and will provide essential input for a quantitative model for mitotic spindle assembly and function.

Acknowledgments We thank Lukas Kapitein and Erwin Peterman for axoneme purification, Maarten Noom, Joost van Mameren, Karen Vermeulen and Daisuke Mizuno for software development, and Russell Stewart for the kinesin plasmid. We also thank Tarun Kapoor and Benjamin Kwok for the Eg5 protein. Support for this work was provided by the Foundation for Fundamental Research on Matter (FOM) (C.F.S.), the DFG Center Molecular Physiology of the Brain (C.F.S. and S.L.) and a Research Grant from the Human Frontier Science Program (C.F.S. and S.L.).

\section{References}

Allersma MW, Gittes F, deCastro MJ, Stewart RJ, Schmidt CF (1998) Two-dimensional tracking of NCD motility by back focal plane interferometry. Biophys J 74:1074-1085

Compton DA (2000) Spindle assembly in animal cells. Annu Rev Biochem 69:95-114

Crevel I, Lockhart A, Cross RA (1997) Kinetic evidence for low chemical processivity in NCD and Eg5. J Mol Biol 273:160 170

Drummond DR, Hagan IM (1998) Mutations in the bimC box of Cut7 indicate divergence of regulation within the bimC family of kinesin related proteins. J Cell Sci 111:853-865

Gibbons IR, Fronk E (1979) Latent adenosine-triphosphatase form of Dynein-1 from Sea-Urchin sperm flagella. J Biol Chem 254:187-196

Gittes F, Schmidt CF (1998a) Interference model for back-focalplane displacement detection in optical tweezers. Optics Lett 23:7-9

Gittes F, Schmidt CF (1998b) Signals and noise in micromechanical measurements. Methods Cell Biol 55:129-156

Grill SW, Kruse K, Julicher F (2005) Theory of mitotic spindle oscillations. Phys Rev Lett 94:108104

Hildebrandt ER, Hoyt MA (2000) Mitotic motors in Saccharomyces cerevisiae. Biochim Biophys Acta 1496:99-116

Kapitein LC, Peterman EJ, Kwok BH, Kim JH, Kapoor TM, Schmidt CF (2005) The bipolar mitotic kinesin Eg5 moves on both microtubules that it crosslinks. Nature 435:114-118

Kapoor TM, Mitchison TJ (2001) Eg5 is static in bipolar spindles relative to tubulin: evidence for a static spindle matrix. J Cell Biol 154:1125-1133

Kashina AS, Baskin RJ, Cole DG, Wedaman KP, Saxton WM, Scholey JM (1996) A bipolar kinesin. Nature 379:270-272

Kwok BH, Kapitein LC, Kim JH, Peterman EJ, Schmidt CF, Kapoor TM (2006) Allosteric inhibition of kinesin-5 modulates the processive directional motility. Nat Chem Biol 2:480-485 
Kwok BH, Yang JG, Kapoor TM (2004) The rate of bipolar spindle assembly depends on the microtubule-gliding velocity of the mitotic kinesin Eg5. Curr Biol 14:1783-1788

Miyamoto DT, Perlman ZE, Burbank KS, Groen AC, Mitchison TJ (2004) The kinesin Eg5 drives poleward microtubule flux in Xenopus laevis egg extract spindles. J Cell Biol 167:813-818

Sawin KE, Leguellec K, Philippe M, Mitchison TJ (1992) Mitotic spindle organization by a plus-end-directed microtubule motor. Nature 359:540-543

Sharp DJ, Rogers GC, Scholey JM (2000) Microtubule motors in mitosis. Nature 407:41-47
Svoboda K, Block SM (1994) Force and velocity measured for single kinesin molecules. Cell 77:773-784

Svoboda K, Schmidt CF, Schnapp BJ, Block SM (1993) Direct observation of kinesin stepping by optical trapping interferometry. Nature 365:721-727

Valentine MT, Fordyce PM, Krzysiak TC, Gilbert SP, Block SM (2006) Individual dimers of the mitotic kinesin motor Eg5 step processively and support substantial loads in vitro. Nat Cell Biol 8:470-476 